\title{
Colorectal Cancer Screening Rates Increased after Exposure to the Patient-Centered Medical Home $(\mathrm{PCMH})$
}

\author{
Beverly B. Green, MD, MPH, Melissa L. Anderson, MS, Jessica Chubak, PhD, \\ Laura Mae Baldwin, MD, MPH, Leah Tuzzio, MPH, Sheryl Catz, PhD, \\ Alison Cole, MD, MPH, and Sally W. Vernon, PhD
}

Objective: The patient-centered medical home (PCMH) includes comprehensive chronic illness and preventive services, including identifying patients who are overdue for colorectal cancer screening (CRCS). The association between PCMH implementation and CRCS during the Systems of Support to Increase Colorectal Cancer Screening Trial (SOS) is described.

Methods: The SOS enrolled 4664 patients from 21 clinics from August 2008 to November 2009. Patients were randomized to usual care, mailed fecal kits, kits plus brief assistance, or kits plus assistance and navigation. A PCMH model that included a workflow for facilitating CRCS was implemented at all study clinics in late 2009. Patients enrolled early had little exposure to the PCMH, whereas patients enrolled later were exposed during most of their first year in the trial. Logistic regression models were used to assess the association between PCMH exposure and CRCS.

Results: Usual care patients with $\geq 8$ months in the PCMH had higher CRCS rates than those with $\leq 4$ months in the PCMH (adjusted difference, 10.1\%; 95\% confidence interval, 5.7-14.6). SOS interventions led to significant increases in CRCS, but the magnitude of effect was attenuated by exposure to the PCMH $(P$ for interaction $=.01)$.

Conclusion: Exposure to a PCMH was associated with higher CRCS rates. Automated mailed and centrally delivered stepped interventions increased CRCS rates, even in the presence of a PCMH. (J Am Board Fam Med 2016;29:191-200.)

Keywords: Cancer Screening, Colon Cancer, Medical Home

Colorectal cancer (CRC) is the second leading cause of cancer death in the United States. ${ }^{1}$ Better treatments have improved survival rates, but morbidity and mortality could be reduced more rapidly

This article was externally peer reviewed.

Submitted 8 September 2015; revised 25 November 2015; accepted 7 December 2015.

From Group Health Permanente, Seattle WA (BBG, LT); the Group Health Research Institute, Seattle WA (BBG, MLA, JC, SC); the University of Washington School of Medicine, Seattle (BBG, LMB, AC); the University of Washington School of Public Health, Seattle (JC); the University of California-Davis Betty Irene Moore School of Nursing, Sacramento (SC); and the University of Texas, Houston, School of Public Health, Houston (SWV).

Funding: This study was funding by the National Cancer Institute of the National Institutes of Health (grant no. R01CA121125).

Conflict of interest: none declared.

Corresponding author: Beverly B. Green, MD, MPH, Group Health Research Institute, 1730 Minor Ave, Seattle, WA 98101 (E-mail: green.b@ghc.org). and cost-effectively by achieving higher uptake and long-term adherence to CRC screening (CRCS). ${ }^{2}$ The patient-centered medical home $(\mathrm{PCMH})$ has generated national attention as a means of improving evidence-based care, including increasing appropriate uptake of US Preventive Task Forcerecommended CRCS tests.

The PCMH was designed to replace episodic care based on illness and patient complaints to provide ongoing "whole-person" comprehensive chronic illness and preventive care services. ${ }^{3} \mathrm{Key}$ domains of the PCMH include (1) team-based care (with the primary care physician leading a team that collectively takes responsibility for the patient's care); (2) adoption of Chronic Care Model practice systems design ${ }^{4}$ (with integration of the Chronic Care Model components leading to engaged patients and prepared practice teams); 
Figure 1. Systems of support to increase colorectal cancer screening (SOS) and patient-centered medical home timeline, August 2008 to November 2010.

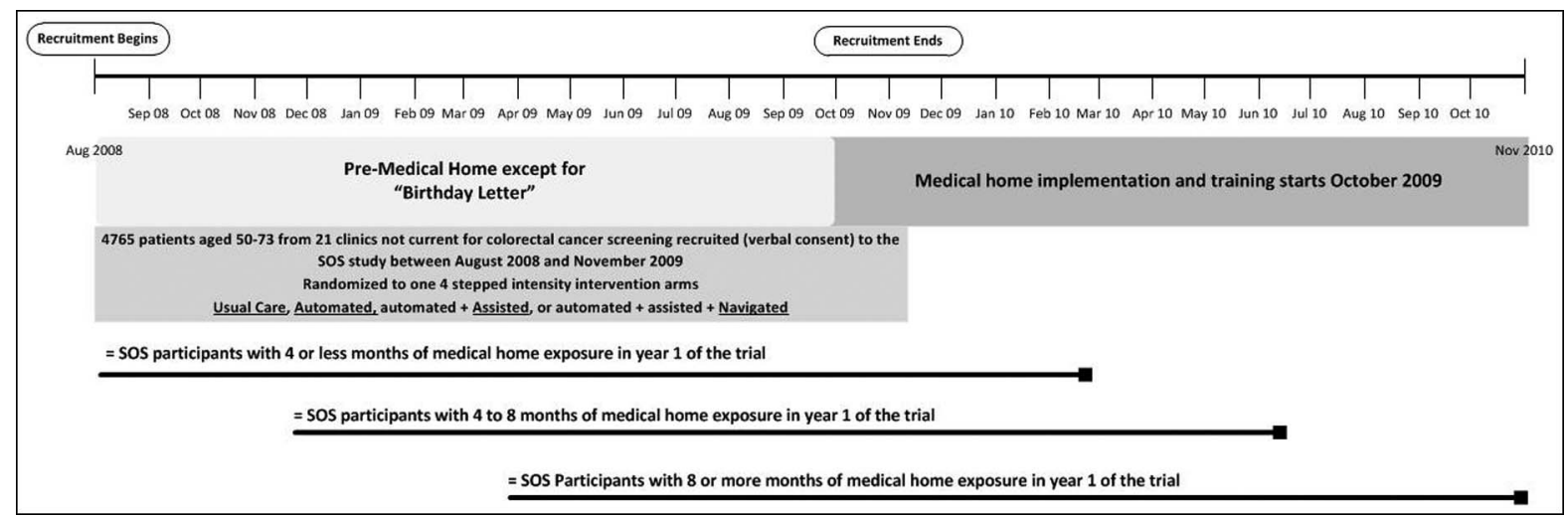

(3) advanced information technologies to support patients and teams providing care; and (4) alignment of payment structures to support and incentivize patient-centered quality care and safety. In support, the National Committee for Quality Assurance has developed a tiered set of PCMH recognition standards. ${ }^{5}$

Observational studies suggest that adoption of the PCMH increases cancer screening rates $^{6}$; the evidence is limited, however, with only 1 study reporting a change in CRCS rates. ${ }^{7}$ We previously demonstrated that a low-cost, health information technology-facilitated mailed intervention with stepped increases in support doubled CRCS uptake and adherence rates over both years of the Systems of Support to Improve Colorectal Cancer Screening and Follow-up trial (SOS). ${ }^{8}$ The SOS took place in an integrated health care organization, Group Health, in Washington State. During the study, Group Health implemented a PCMH in all its primary care clinics, ${ }^{9}$ including the 21 clinics in Western Washington where SOS participants received their care. The PCMH rollout occurred at the end of SOS patient recruitment (October 2009 to January 2010). The coincidental conduct of the SOS at the same time that the PCMH was being implemented in clinics provided an opportunity to study the relationship between the PCMH and adherence to recommended CRCS. Because SOS recruitment was spread out over 16 months (August 2008 to November 2009), study participants had varying exposure to PCMH team-based care: some patients had little or no exposure in year 1 , and for some with their year 1 trial enrollment occurred entirely or almost entirely after the $\mathrm{PCMH}$ was implemented (Figure 1).

PCMH CRCS activities were implemented by clinic staff, whereas SOS interventions were delivered centrally by the study team. We hypothesized that (1) CRCS rates would be higher among SOS usual care group participants with more exposure to the PCMH in year 1 of their study participation compared with those with less exposure, and (2) the PCMH would attenuate the benefit of the SOS intervention, but automated interventions would still be more effective than usual care. This analysis compares CRCS by level of PCMH exposure and examines the interaction between clinic-based $\mathrm{PCMH}$ interventions and centralized SOS interventions.

\section{Methods}

The SOS was supported by the National Cancer Institute (grant no. R01CA121125). The study had institutional review board approval, and written informed consent was not required. Methods, recruitment, and results of the 2-year parent study have been previously published. ${ }^{8,10-13}$ This study is registered at ClinicalTrials.gov (identifier NCT00697047).

\section{Study Participants}

Participants aged 50 to 73 years were recruited between August 2008 and November 2009 from 21 Group Health medical centers (clinics) in western Washington State. Group Health is a large, nonprofit integrated health care delivery system in the Pacific Northwest. Patients were identified using 
electronic health records (EHRs) and were eligible if they were not current or were soon due for CRCS, defined as no colonoscopy within 9 years, no flexible sigmoidoscopy (sigmoidoscopy) within 4 years, or no fecal test within 9 months. Patients with a prior CRC diagnosis, with inflammatory bowel disease, or with end-stage or life-threatening disease (eg, dementia, renal failure) were excluded. Potential participants were mailed a letter about the study and were then called to confirm eligibility and to obtain verbal consent.

Participants $(\mathrm{N}=4664)$ were randomized to receive either (1) usual care or 1 of 3 stepped-care interventions; (2) an EHR-linked automated mailed program that included information on CRCS choice, a number to call for endoscopy (colonoscopy or sigmoidoscopy), and mailed fecal kit for those not calling ("automated"); (3) automated plus brief phone assistance from a medical assistant (MA) to identify their test preference (endoscopy or fecal kit) and to complete their CRCS test choice ("assisted"); or (4) assisted plus ongoing support from a nurse navigator for overcoming screening barriers ("navigated").

\section{Interventions}

The interventions associated with the 4 arms of the SOS listed above have been previously described in detail $^{11}$ and are summarized briefly here.

\section{Usual Care}

All participants received usual care, which at Group Health includes an annual birthday letter from their physician with the date of their last CRCS test, general guidelines for testing intervals, and a message to contact the physician unless "you know you are current on screening." Group Health guidelines for CRCS paralleled those recommended by the US Preventive Services Task Force. Patients randomized to usual care received standard care, including the PCMH interventions described below. Since SOS participants were recruited from August 2008 to November 2009, about half were exposed to the PCMH in the first year of the SOS and all were exposed in year 2 (Figure 1).

\section{Stepped Interventions}

Patients randomized to the active intervention groups received usual care plus 1 of 3 stepped interventions: automated interventions ("auto- mated"), automated plus assisted care ("assisted"), or automated and assisted plus navigated care ("navigated").

Automated interventions used an EHR-linked study registry to track when CRCS was due and to automatically generate mailings. Patients due for CRCS received an informational pamphlet about the different CRCS options (ie, fecal tests, sigmoidoscopy, and colonoscopy). Patients were informed that fecal kits (Hemoccult SENSA; Beckman Coulter, Brea, CA) would be mailed, but that they could call the SOS phone line if they preferred another type of screening test (few patients called). Noncallers (the vast majority) were mailed fecal kits with simplified pictorial instructions and a postagepaid return envelope. Patients without EHR evidence of a completed CRCS test received a reminder letter after 3 weeks.

Assisted patients received usual care and the automated mailings plus brief telephone assistance delivered by an MA if they had not completed CRCS. MAs used the study registry to view lists of patients who had called to request alternate screening or had not completed fecal testing within 3 weeks of the mailing. MAs asked patients about their CRCS choice and provided brief assistance to facilitate completion (eg, if the patient preferred sigmoidoscopy or colonoscopy, the MA forwarded the request to the patient's physician and recontacted the patient with further instructions).

In addition to usual care, automated, and assisted support, navigated patients not completing CRCS after automated and assisted interventions received additional support from a registered nurse (RN). RN care included assessing patients' CRC risk, reviewing procedural risk, providing motivational counseling to assist patients in defining their screening intent, creating a patient-shared screening action plan, assisting with referrals and endoscopy preparation, and tracking testing completion. If the $\mathrm{RN}$ could not reach the patient, a letter or secure E-mail reiterating the importance of CRCS was sent; it included the RN's phone number for further assistance.

Mailed interventions were implemented centrally by SOS staff. MAs and nurses who conducted the interventions were employed by the organization and had protected time to deliver the interventions to patients from all 21 clinics. 
Figure 2. Patient-centered medical home process map for medical assistant/nurse (MA) in-reach and outreach colorectal cancer screening (CRCS) processes.

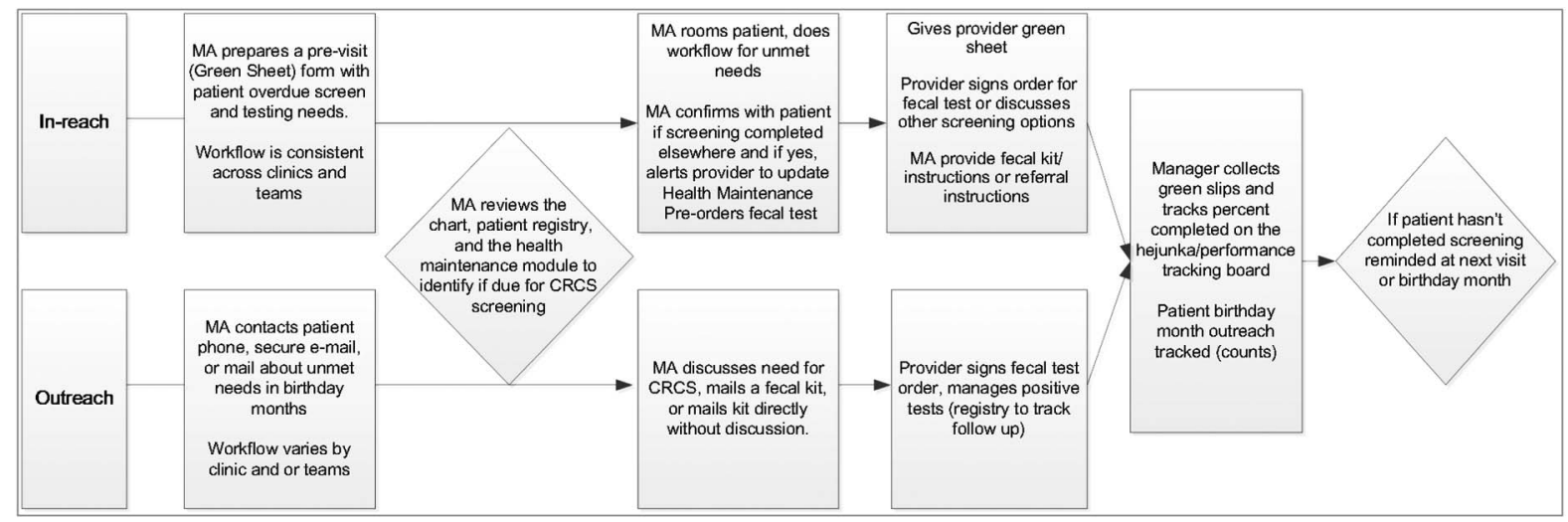

\section{The PCMH at Group Health}

The PCMH implemented at Group Health includes clinic-based interventions to improve prevention and chronic disease care. The PCMH model emphasizes team-based care, which includes MAs or nurses using an organization-wide registry to identify patients' preventive and chronic care needs (eg, immunizations, $\mathrm{HbA}_{1 \mathrm{c}}$ for patients with diabetes) before scheduled visits, and offering these services at clinic visits ("in-reach"; Figure 2). This in-reach includes MAs or nurses identifying patients who are overdue for CRCS testing and providing them with fecal test kits or placing an order for endoscopy for the provider to approve, depending on the patient's preference. In addition, during patients' birthday months, MAs/nurses use the registry to identify care needs and remind patients of needed services by phone, secure E-mail, or mail (“outreach"; Figure 2). Outreach included mailing fecal test kits to patients due for CRCS. PCMH training started in October 2009, ${ }^{9}$ with the exception of 1 primary care clinic that implemented the PCMH in 2007 to pilot and refine PCMH components. Physicians received financial incentives for meeting organizational service (eg, access, patient satisfaction) and quality of care objectives (chronic disease and prevention metrics, including CRCS).

All primary care clinics completed PCMH training by January 2010 and were expected to conduct in-reach and outreach procedures, including those specific to CRCS, as part of standard clinical care thereafter. All were recognized as National Committee for Quality Assurance level 3 PCMHs (ie, compliance with all $10 \mathrm{PCMH}$ elements and performance measures) by October $2010 .^{14}$

\section{Key Stakeholder Interviews}

To understand and describe how the PCMH was implemented with respect to CRCS, the principal investigator (BBG) and a project manager (LT) visited 12 of the 21 Group Health PCMH clinics where SOS participants received care. A staff survey was conducted in October 2010 to evaluate the first year of PCMH implementation, which included a question on employee satisfaction with Group Health as a place to work. ${ }^{9}$ Based on the advice of these researchers, we randomly selected 6 clinics with high and 6 clinics with low employee satisfaction to receive site visits. BBG and LT were blinded to clinic score results. An administrative and clinical leader and 2 MAs or nurses were interviewed at each site. Interview topics included how CRCS in-reach and outreach processes were implemented in the PCMH. This included discussing the specific workflows and tools used (eg, before the visit, MA's use of the registry and chart review to identify patients overdue for CRCS, use of mailings and other reminders for outreach), monitoring of PCMH workflow activities, and assessment of the PCMH rollout in general and with respect to CRCS in particular. We also briefly toured the clinic to observe any apparent approaches or tools the clinic used to support their CRCS efforts. Interview and observational data were recorded in field notes, and content was summarized to describe how the PCMH was implemented across clinics.

\section{Exposure to the PCMH}

For this analysis, the primary exposure was months of exposure to the PCMH in year 1 of the SOS. The level of exposure to the PCMH in year 1 was based on the patient's SOS randomization date and 
the date of PCMH implementation at the patient's clinic. For example, a patient recruited early in the study (between August and October 2008) would have no exposure to the PCMH because no clinics rolled out the PCMH before October 2009. Patients recruited late in 2009 would be exposed to the PCMH for all or almost all of the year 1 study period because all clinics had implemented the PCMH by January 2010. SOS participants were categorized into 1 of 3 groups by exposure to the PCMH in year 1: (1) $\leq 4$ months, $(2)>4$ but $<8$ months, and (3) $\geq 8$ months. Analyses followed an intent-to-treat-like approach, with exposure defined by randomization and PCMH implementation dates, regardless of the actual exposure to $\mathrm{PCMH}$ outreach and in-reach activities received by the individual participants. For example, a participant would be classified as high exposure if $\mathrm{PCMH}$ implementation was in place in their clinic at the time of SOS randomization; however, they may not have actually received clinic-based outreach or inreach activities if they did not have any clinic visits or were screened early and therefore did not trigger PCMH activities.

\section{CRC Testing Outcomes}

The primary outcome of any CRC test completion was defined as evidence in the EHR or claims data of completion of any of the following tests in year 1: fecal testing, sigmoidoscopy, or colonoscopy. Secondary outcomes included the type of screening test completed (ie, fecal test or endoscopy). Since the data sources used to define these outcomes did not contain sufficient information to determine whether a test was screening or diagnostic, outcome measures were based on evidence of CRC testing regardless of indication.

\section{Statistical Analyses}

Logistic regression models were used to evaluate the association between exposure to the PCMH and CRC testing. To estimate the association between PCMH and CRC testing in the absence of the SOS stepped interventions, the analysis was restricted to participants who were randomized to the usual care group. Models were adjusted for age, sex, race, education, and prior CRC testing. Robust standard error estimation accounted for correlation between patients within the same clinic. The unadjusted percentage who received CRC testing by $\mathrm{PCMH}$ exposure group is reported. Calculated predictive margins are based on the logistic regression model estimates. ${ }^{15}$ Predictive margins were used to estimate adjusted relative risks and risk differences of completing CRC testing, comparing those with moderate exposure (4 to 8 months) and high exposure to the PCMH ( 8 or more months) with the referent group (those with low exposure [ $\leq 4$ months]).

To determine whether the effect of the SOS intervention differed by level of exposure to the PCMH, a similar logistic regression model was used, which included main effects for both PCMH exposure and SOS intervention group (usual care vs any SOS intervention) and their interaction. This analysis included all SOS participants. Unadjusted probabilities, and adjusted relative risks and risk differences of CRC testing in the combined SOS intervention group, were compared with usual care (referent group) within strata defined by level of exposure to the PCMH.

Patients receiving care at the pilot site were included in the main analysis, but sensitivity analyses excluded the pilot clinic, which did not differ from the main results and are not presented here. Analyses were completed using Stata statistical software, version 13.0 (Stata Corp., College Station, TX).

\section{Results}

Of the 4664 SOS participants, $30 \%$ had $<4$ months of PCMH exposure, $27 \%$ had between $>4$ and $<8$ months, and $43 \%$ had $>8$ months (Table 1 ). Because randomization in the parent trial was stratified by clinic and the PCMH was implemented by clinic, exposure to the PCMH was balanced across SOS intervention groups. Patient characteristics were generally similar across groups, except for whether participants had completed any type of CRC testing before study enrollment.

Usual care participants exposed to the PCMH for at least 8 months had higher CRC testing rates than those with less exposure (adjusted difference, 10.1\%; 95\% CI, 5.7-14.6) (Table 2). Increased rates of testing were the result of increased completion of fecal tests (adjusted difference, 12.1\%; 95\% CI, 5.9-18.2).

SOS active interventions were more effective than usual care regardless of PCMH exposure level (Table 3), but the effect size was attenuated with greater exposure to the PCMH. The adjusted dif- 
Table 1. Participant Characteristics By Assignment to Usual Care or Active Interventions and Exposure to a Patient-Centered Medical Home in Year 1 of the Systems of Support to Increase Colorectal Cancer Screening Trial

\begin{tabular}{|c|c|c|c|c|c|c|}
\hline \multirow{3}{*}{$\begin{array}{l}\text { Characteristics of Exposure } \\
\text { Group }\end{array}$} & \multicolumn{6}{|c|}{ SOS Randomization Group By Duration of Exposure to a Medical Home (Months) } \\
\hline & \multicolumn{3}{|c|}{ SOS Usual Care } & \multicolumn{3}{|c|}{ SOS Intervention } \\
\hline & $\leq 4$ & $>4$ and $<8$ & $\geq 8$ & $\leq 4$ & $>4$ and $<8$ & $\geq 8$ \\
\hline Participants & $351(30.1)$ & $316(27.1)$ & $499(42.8)$ & $1048(30.0)$ & $935(26.7)$ & $1515(43.3)$ \\
\hline \multicolumn{7}{|l|}{ Age at randomization (years) } \\
\hline $50-64$ & $299(85.2)$ & $265(83.9)$ & $428(85.8)$ & $895(85.4)$ & $784(83.9)$ & $1303(86.0)$ \\
\hline $65-73$ & $52(14.8)$ & $51(16.1)$ & $71(14.2)$ & $153(14.6)$ & $151(16.2)$ & $212(14.0)$ \\
\hline Female sex & $196(55.8)$ & $168(53.2)$ & $289(57.9)$ & $570(54.4)$ & $510(54.6)$ & $811(53.5)$ \\
\hline \multicolumn{7}{|l|}{ Race/ethnicity } \\
\hline Black & $17(4.9)$ & $12(3.8)$ & $15(3.1)$ & $57(5.5)$ & $51(5.5)$ & $76(5.0)$ \\
\hline Asian & $28(8.0)$ & $14(4.5)$ & $22(4.5)$ & $56(5.4)$ & $47(5.1)$ & $70(4.6)$ \\
\hline Hispanic & $8(2.3)$ & $15(4.9)$ & $20(4.1)$ & $25(2.4)$ & $28(3.0)$ & $58(3.8)$ \\
\hline Non-Hispanic white & $282(80.8)$ & $261(83.1)$ & $407(82.7)$ & $845(81.0)$ & $738(79.5)$ & $1203(79.6)$ \\
\hline Other & $14(4.0)$ & $12(3.8)$ & $28(5.7)$ & $60(5.8)$ & $64(6.9)$ & $104(6.9)$ \\
\hline \multicolumn{7}{|l|}{ General health } \\
\hline Excellent/very good & $221(63.1)$ & $184(58.2)$ & $317(63.7)$ & $676(64.7)$ & $565(60.4)$ & $972(64.2)$ \\
\hline Good & $105(30.0)$ & $100(31.7)$ & $133(26.7)$ & $303(29.0)$ & $298(31.9)$ & $452(29.9)$ \\
\hline Fair/poor & $24(6.9)$ & $32(10.1)$ & $48(9.6)$ & $66(6.3)$ & $72(7.7)$ & $90(5.9)$ \\
\hline $\begin{array}{l}\text { Married or living with a } \\
\text { partner }\end{array}$ & $253(72.3)$ & $224(71.1)$ & $360(72.3)$ & $772(73.9)$ & $704(75.4)$ & $1126(74.3)$ \\
\hline \multicolumn{7}{|l|}{ Highest education } \\
\hline $\begin{array}{l}\text { High school } \\
\text { graduate/GED or less }\end{array}$ & $51(14.5)$ & $62(19.7)$ & $78(15.7)$ & $121(11.6)$ & 155 (16.6) & $233(15.4)$ \\
\hline Some college & $112(31.9)$ & $107(34.0)$ & $149(29.9)$ & $315(30.1)$ & $322(34.5)$ & $462(30.5)$ \\
\hline Bachelor's degree or higher & $188(53.6)$ & $146(46.4)$ & $271(54.4)$ & $611(58.4)$ & $457(48.9)$ & $820(54.1)$ \\
\hline \multicolumn{7}{|l|}{ Smoking status } \\
\hline Current & $36(10.4)$ & $56(18.2)$ & $62(12.7)$ & $117(11.4)$ & $99(10.8)$ & $175(11.8)$ \\
\hline Former & $92(26.5)$ & $80(26.0)$ & $137(28.1)$ & $299(29.1)$ & $262(28.5)$ & $426(28.8)$ \\
\hline Never & $219(63.1)$ & $172(55.8)$ & $288(59.1)$ & $611(59.5)$ & $557(60.7)$ & $880(59.4)$ \\
\hline $\begin{array}{l}\text { Never tested for CRC (before } \\
\text { randomization) }\end{array}$ & $146(41.6)$ & $153(48.4)$ & $240(48.1)$ & $438(41.8)$ & $433(46.3)$ & $750(49.5)$ \\
\hline $\begin{array}{l}\text { First-degree relative with } \\
\text { CRC }\end{array}$ & $18(5.2)$ & $17(5.5)$ & $20(4.1)$ & $57(5.5)$ & $45(4.9)$ & $58(3.9)$ \\
\hline
\end{tabular}

Data collection occurred between 2008 and 2009. Data are n (\%).

CRC, colorectal cancer; SOS, Systems of Support to Increase Colorectal Cancer Screening Trial.

ference in the proportion tested between the combined intervention arms and usual care was $34.4 \%$ (95\% CI, 30.6-38.2) with PCMH exposure of $\leq 4$ months, $34.1 \%$ (95\% CI, 27.4-40.8) for $>4$ to $<8$ months of PCMH exposure, and $21.9 \%$ (95\% CI, 17.4-26.4) with exposure of $\geq 8$ months of PCMH exposure ( $P=.01$, test for interaction). Increased rates of testing were the result of fecal tests. Effect attenuation was not influenced by intervention intensity (automated, assisted, navigated stepped arm assignment; data not shown).

Interviews with clinic stakeholders provided information on PCMH implementation. There was little variation in descriptions of CRCS-re- lated workflow for in-reach processes within and across clinics. During the clinic visits, MAs/ nurses directly demonstrated their process for reviewing CRCS status and providing fecal kits or an endoscopy order at clinic visits for patients overdue for screening, as well as for documentation of these activities (Figure 2). Clinic administrators described $\mathrm{PCMH}$ in-reach as "standard work" and showed us the visual poster board charts that were used to track MA/nurse completion of previsit tasks (checking off a worksheet of patient prevention and chronic care needs). There was greater variation among MA/nurse and clinic administrator reports of outreach ac- 
Table 2. Colorectal Cancer Screening Uptake* among Patients Receiving Usual Care in the Systems of Support to Increase Colorectal Cancer Screening Trial, by Exposure to a Patient-Centered Medical Home

\begin{tabular}{|c|c|c|c|}
\hline \multirow[b]{2}{*}{ Usual Care Only } & \multicolumn{3}{|c|}{ Duration of Exposure to Medical Home (months) } \\
\hline & $\begin{array}{c}\leq 4 \\
(\mathrm{n}=351)\end{array}$ & $\begin{array}{c}>4 \text { and }<8 \\
(\mathrm{n}=316)\end{array}$ & $\begin{array}{c}\geq 8 \\
(\mathrm{n}=499)\end{array}$ \\
\hline \multicolumn{4}{|l|}{ Primary outcome } \\
\hline \multicolumn{4}{|l|}{ Any CRCS* } \\
\hline Unadjusted percent (95\% CI) & $36.8(31.9-41.9)$ & $34.8(29.7-40.2)$ & $44.9(40.6-49.3)$ \\
\hline Adjusted $\mathrm{RR}^{\dagger}(95 \% \mathrm{CI})$ & 1.00 (Referent) & $0.99(0.81-1.18)$ & $1.29(1.15-1.42)$ \\
\hline Adjusted difference ${ }^{\ddagger}(95 \% \mathrm{CI})$ & Referent & $-0.2(-6.9$ to 6.5$)$ & $10.1(5.7-14.6)$ \\
\hline \multicolumn{4}{|l|}{ Secondary outcomes } \\
\hline \multicolumn{4}{|l|}{ Any fecal test ${ }^{\S}$} \\
\hline Unadjusted percentage (95\% CI) & $21.1(17.1-25.7)$ & $20.6(16.5-25.4)$ & $32.7(28.7-36.9)$ \\
\hline Adjusted $\mathrm{RR}^{\dagger}(95 \% \mathrm{CI})$ & 1.00 (Referent) & $0.99(0.64-1.35)$ & $1.58(1.22-1.94)$ \\
\hline Adjusted difference $^{\ddagger}(95 \% \mathrm{CI})$ & Referent & $-0.1(-7.6$ to 7.3$)$ & $12.1(5.9-18.2)$ \\
\hline \multicolumn{4}{|l|}{ Any endoscopy } \\
\hline Unadjusted percentage (95\% CI) & $21.4(17.4-26.0)$ & $20.6(16.5-25.4)$ & $18.2(15.1-21.9)$ \\
\hline Adjusted $\mathrm{RR}^{\dagger}$ (95\% CI) & 1.00 (Referent) & $1.03(0.77-1.29)$ & $0.95(0.73-1.18)$ \\
\hline Adjusted difference $^{\ddagger}(95 \% \mathrm{CI})$ & Referent & $0.6(-4.5$ to 5.8$)$ & $-0.9(-5.4$ to 3.6$)$ \\
\hline
\end{tabular}

*Any colorectal cancer screening (CRCS) test included completion of fecal occult blood tests, flexible sigmoidoscopy, or colonoscopy during year 1 of the Systems of Support to Increase Colorectal Cancer Screening Trial (SOS). All patients were overdue for CRCS. ${ }^{+}$Relative risk (RR) of screening in the exposure group relative to the group with $\leq 4$ months of exposure to a patient-centered medical home (PCMH), adjusted for age, sex, race, education, and prior CRCS.

${ }^{\ddagger}$ Adjusted difference is the percentage screened in the exposure group minus the percentage screened in group with $\leq 4$ months of exposure to a PCMH, adjusted for age, sex, race, education, and prior CRCS.

${ }^{\S}$ Hemoccult SENSA.

CI, confidence interval.

tivities during patients' birthday months. Some MAs/nurses called or sent letters notifying patients they were overdue and sent kits if patients requested them. Other MAs/nurses described mailing kits without notifying the patient first. The effects of PCMH exposure on CRCS rates were similar across clinics with high, low, or average satisfaction with Group Health as a place to work (data not shown).

\section{Discussion}

Our study provides further evidence that implementation of the PCMH model of care leads to increased uptake of CRCS. ${ }^{7}$ The PCMH emphasized clinic-based procedures to increase CRCS. Increases in CRCS uptake were almost entirely the result of increased fecal testing in both the usual care and intervention groups. This is not surprising because both PCMH and SOS interventions emphasized fecal testing. As part of the PCMH, MAs preorder fecal kits and provide these directly to patients at clinic visits or by mail as part of outreach. SOS interventions mailed fecal kits to everyone, except for the few who called to request an alternative test. Even when usual care included a fully implemented PCMH, stepped centralized SOS interventions were still more effective than usual care, but the magnitude of benefit was less.

It is important to note that SOS interventions were delivered centrally, with patients getting the same intervention across multiple clinics, whereas $\mathrm{PCMH}$ interventions were implemented within clinics. We previously indicated that the centralized approach saves costs (for the automated-only and automated-plus-assisted arms), and costs are quite reasonable for the full-intensity intervention arm (automated plus assisted and navigated). ${ }^{10}$ This has implications for health care systems and their organization of care in a PCMH. Patients often have multiple preventive and chronic care needs, and outsourcing 1 activity to a centralized service might allow teams to focus more attention on other care needs.

Of note, in key stakeholder interviews we found remarkable uniformity among clinic in-reach 


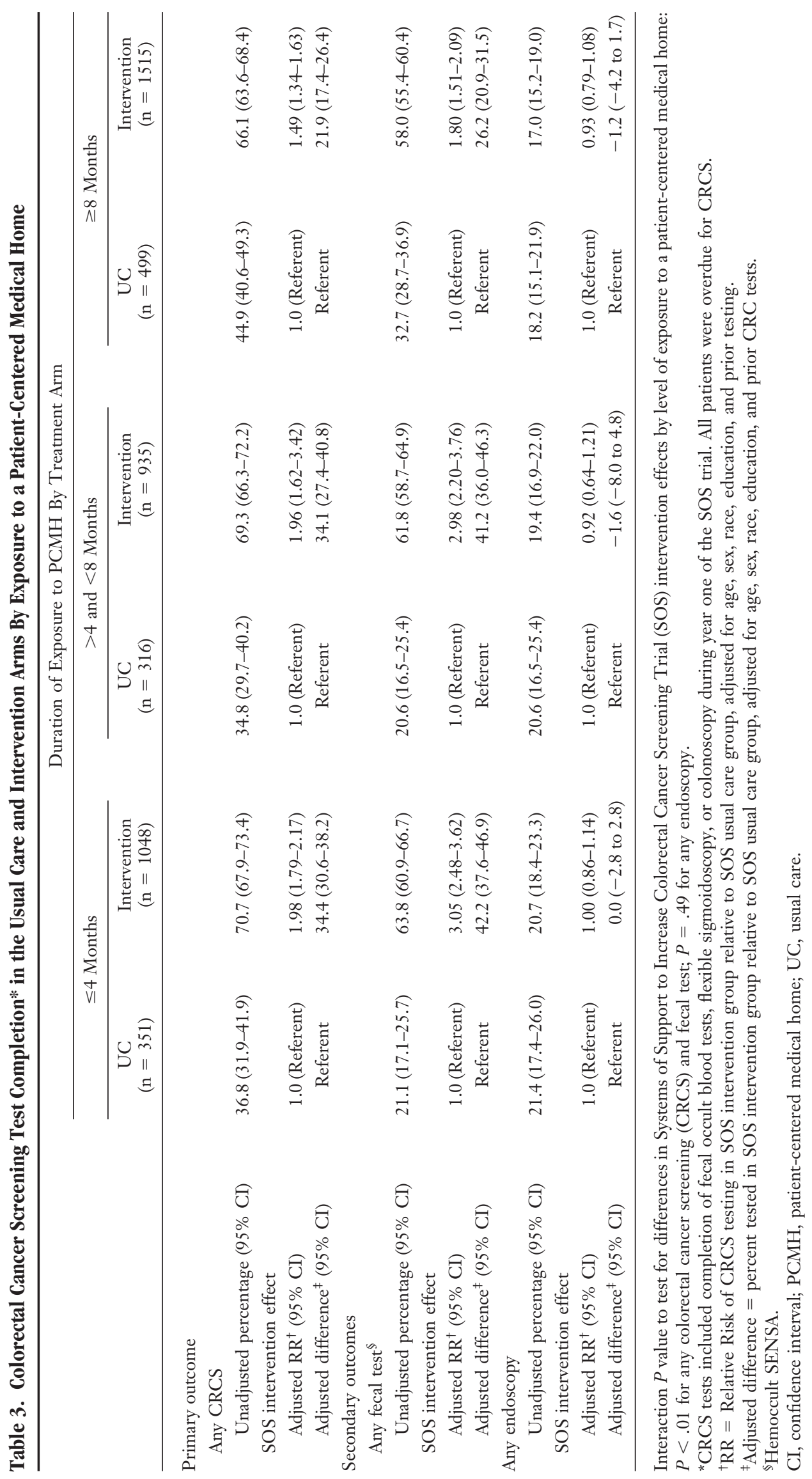

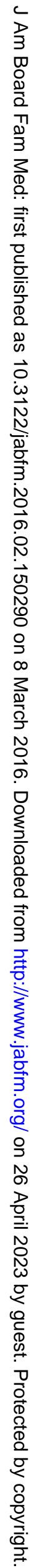


CRCS promotion activities across sites. This mirrors results from formal evaluations of the Group Health PCMH implementation experience., ${ }^{9} 16$ Group Health was able to roll out the PCMH quite rapidly; $80 \%$ to $100 \%$ of clinics met implementation targets by 12 months for most key process measures, including $100 \%$ of visits with previsit documentation preparation by MAs. However, CRCS outreach processes were more varied. This might be because primary care practice is more geared toward face-to-face visits, and out-of-office care activities had to be squeezed into the day-today routine. In a study of community clinics that had adopted a PCMH, there was inconsistent use of these systems and a lack of policies and workflows to assure appropriate follow-up of positive screening tests and diagnostic results, even among clinics with systems to track CRCS. ${ }^{17,18}$ In addition, the SOS automated system tracked when patients were due for CRCS, whereas visits and birthday reminders paired with outreach might not be in sync with the time that the patient is due for screening, particularly fecal testing, which is recommended annually.

Our study has limitations. Although the parent study was a randomized controlled trial, this analysis was observational in nature. That is, the level of $\mathrm{PCMH}$ exposure was not randomized. Exposure level was associated with both calendar time and clinic, with the possibility of residual confounding; that is, temporal changes in screening rates or unmeasured differences across clinic populations could have influenced the results. In addition, these findings may not be generalizable to all populations. Study participants provided consent and, as previously published, were more likely to have higher levels of education, to be non-Hispanic white, and to have previously completed a CRCS test. ${ }^{12}$ In addition, the SOS took place in an integrated group practice and had resources other clinics might not have, including a comprehensive EHR, registries, and the ability to capture both CRC clinical and claims testing data (eg, colonoscopies occurring outside of the health system but paid for by the insurance plan). These resources are critical components of both the centralized SOS program and clinic $\mathrm{PCMH}$ efforts to increase CRCS. Finally, the SOS design allowed us to look at only 1 year of PCMH implementation, and it is possible that CRCS uptake as part of PCMH usual care improved further over time.

\section{Conclusion}

As hypothesized, implementation of a PCMH team care model that included clinic-based in-reach and outreach was associated with higher CRC screening rates among SOS usual care group participants. SOS interventions led to significant increases in CRCS uptake, but the magnitude of the effect was inversely related to the level of PCMH exposure. Adding centralized CRCS promotion activities such as those offered by the SOS might reduce some of the burden experienced by primary care in trying to improve population-based care in a PCMH and allow clinic personnel to focus on activities that require in-person services. Future research could study ways to efficiently integrate both programs and further optimize screening rates.

\section{Figure 2: Medical Home Diagram}

\section{Usual Care CRCS Process at Group Health}

The flowchart in Figure 2 illustrates the usual care process for CRCS at Group Health. The standard elements across clinics include having a performance tracking board, access to the EHR screening history and current status, and standard steps to provide outreach to patients and to remind patients who come into the clinic about screening. In general, CRCS is a team process involving the MA/ nurse and provider (physician, physician assistant, or nurse practitioner), particularly to validate the information and submit orders. The MA assesses which patients are due for screening and, depending on when the patient's next visit is to primary care, they either do in-reach activities or reach out. In-reach includes preparing the chart for the visit by including a previsit summary sheet and, if indicated, highlighting that a CRCS test is due. The MA will talk with the patient, and most times the provider will also speak with the patient. It varies from clinic to clinic, but some MAs send reminder letters after the visit. The standard outreach process is done for patients who are not scheduled to come in for a visit but who are due to complete a screening test. The typical process includes the MA contacting the patient either by phone or by using template phrases in a secure E-mail or mailed letter. The mode of contact is based on the MA's preference and is not standardized.

\section{References}

1. Siegel RL, Miller KD, Jemal A. Cancer statistics, 2015. CA Cancer J Clin 2015;65:5-29. 
2. Vogelaar I, van Ballegooijen M, Schrag D, et al. How much can current interventions reduce colorectal cancer mortality in the U.S.? Mortality projections for scenarios of risk-factor modification, screening, and treatment. Cancer 2006;107:162433.

3. Berenson RA, Hammons T, Gans DN, et al. A house is not a home: keeping patients at the center of practice redesign. Health Aff (Millwood) 2008;27: 1219-30.

4. Wagner EH, Austin BT, Von Korff M. Organizing care for patients with chronic illness. Milbank Q 1996;74:511-44.

5. National Committee for Quality Assurance. Patientcentered medical home recognition. Available from: http://www.ncqa.org/Programs/Recognition/Practices/ PatientCenteredMedicalHomePCMH.aspx. Accessed August 23, 2015.

6. Pandhi N, DeVoe JE, Schumacher JR, et al. Preventive service gains from first contact access in the primary care home. J Am Board Fam Med 2011;24: 351-9.

7. Markovitz AR, Alexander JA, Lantz PM, Paustian ML. Patient-centered medical home implementation and use of preventive services: the role of practice socioeconomic context. JAMA Intern Med 2015; 175:598-606.

8. Green BB, Wang CY, Anderson ML, et al. An automated intervention with stepped increases in support to increase uptake of colorectal cancer screening: a randomized trial. Ann Intern Med 2013;158: 301-11.

9. Hsu C, Coleman K, Ross TR, et al. Spreading a patient-centered medical home redesign: a case study. J Ambul Care Manage 2012;35:99-108.

10. Meenan RT, Anderson ML, Chubak J, et al. An economic evaluation of colorectal cancer screening in primary care practice. Am J Prev Med 2015;48: 714-21.

11. Green BB, Wang CY, Horner K, et al. Systems of support to increase colorectal cancer screening and follow-up rates (SOS): design, challenges, and baseline characteristics of trial participants. Contemp Clin Trials 2010;31:589-603.

12. Green BB, Bogart A, Chubak J, et al. Nonparticipation in population-based trial to increase colorectal cancer screening. Am J Prev Med 2012;42:390-7.

13. Murphy CC, Verson SW, Haddock NM, Anderson ML, Chubak J, Green BB. Longitudinal predictors of colorectal cancer screening among participants in a randomized controlled trial. Prev Med 2014;66: 123-30.

14. National Committee for Quality Assurance. Accreditation levels. Available from: http://www.ncqa. org/Programs/Accreditation/AccountableCare OrganizationACO/ACOAccreditationLevels.aspx. Accessed August 23, 2015.

15. Lane PW, Nelder JA. Analysis of covariance and standardization as instances of prediction. Biometrics 1982;38:613-21.

16. Reid RJ, Johnson EA, Hsu C, et al. Spreading a medical home redesign: effects on emergency department use and hospital admissions. Ann Fam Med 2013;11(Suppl 1):S19-26.

17. Sarfaty M, Myers RE, Harris DM, et al. Variation in colorectal cancer screening steps in primary care: basis for practice improvement. Am J Med Qual 2012;27:458-66.

18. Sarfaty M, Stello B, Johnson M, Sifri R, Borsky A, Myers RM. Colorectal cancer screening in the framework of the medical home model: findings from focus groups and interviews. Am J Med Qual 2013;28:422-8. 